\title{
Recursos individuais e contextuais de desenvolvimento pessoal: Adaptação portuguesa do developmental assets profile
}

\author{
Internal and external developmental assets:
}

\section{Portuguese adaptation of developmental assets profile}

\author{
Nuno Archer de Carvalho*, Feliciano Henriques Veiga** \\ * Colégio Pedro Arrupe (Portugal), ** Instituto de Educação da Universidade de Lisboa (Portugal)
}

\begin{abstract}
Resumen
Enquadramento: A valorização de um olhar compreensivo e ecológico do desenvolvimento na adolescência tem dado maior atenção à investigação teórica e empírica dos recursos de desenvolvimento. Objetivos: adaptar à realidade portuguesa a escala Developmental Assets Profile (DAP). Método: tendo por base os procedimentos associados à adaptação psicométrica de instrumentos psicológicos, aplicou-se a escala a uma amostra de 201 alunos do $6^{\circ}, 9^{\circ}$ e $11^{\circ}$ ano, na presença do investigador e com participação consentida. Resultados: verificaram-se especificidades em relação ao instrumento original, tanto ao nível da correspondência fatorial como da consistência interna das dimensões; no estudo da validade externa, verificaram-se correlações significativas com as dimensões de bem-estar psicológico. Conclusões: os resultados encontrados permitiram valorizar a versão portuguesa da escala, justificando-se, contudo, a continuação do seu estudo, no sentido da obtenção de novos instrumentos, cuja solidez teórica e empírica sirva tanto a investigação como a intervenção educativa com jovens.

Palabras clave: desenvolvimento positivo dos jovens, recursos de desenvolvimento, developmental assets profile
\end{abstract}

\begin{abstract}
Context: the attention given to a comprehensive and ecological approach of adolescent development has promoted the research on developmental assets. Goals: adapt to a portuguese sample the Developmental Assets Profile (DAP). Method: regarding the procedures for the psicometric adaptation of psychologic instruments, the scale was used with 201 adolescents of the 6th, 9th and 11 th grades, in the presence of the researcher and with consented participation. Results: the psychometric results found are different from the original results; evidence of significant correlations were observed with psychological wellbeing indicators. Conclusions: despite the value of the Portuguese adaptation here presented, the results demand further work to attain new instruments with theoretical and empirical soundness.
\end{abstract}

Keyword: positive youth development, developmental assets, developmental assets profile.
No seu diário de professor Sebastião da Gama escreve sobre os alunos: "o que eu quero principalmente é que vivam felizes!" (2003, p. 23). Talvez pudesse formular este desejo como a possibilidade de virem a ter "full and productive lives" (Merrell \& Gueldner, 2010). As mudanças sociais, nomeadamente na família (Benson, Leffert, Scales \& Blyth, 2012), o tempo e as experiências vividas na escola, fazem dela um contexto privilegiado de desenvolvimento das crianças e jovens (Gomez \& Ang, 2007).

A adolescência, período que permite à criança entrar na vida adulta, distingue-se pela rapidez, profundidade e intensidade das mudanças, pelo amadurecimento biológico, cognitivo, social e emocional e pela necessária reorganização estrutural e funcional (Bizarro, 1999). É um tempo de muitas escolhas escolares, vocacionais, interesses, ensaio de comportamentos sociais e de intimidade (Simões, 2010), com impacto na vida adulta.

Ao olhar pessimista da adolescência "storm and stress" (Lerner, 2005), de mudanças dramáticas e perigosas, opõe-se o olhar otimista que, sem ignorar os fatores de risco, promove a resiliência (Simões, 2010), o funcionamento positivo e as oportunidades dos jovens para crescer com os desafios (Bizarro, 1999), desenvolverem os seus talentos, forças, competências e interesses (Guerra \& Bradshaw, 2008), para poderem ser adultos motivados, orientados, conscientes, socialmente competentes, compassivos e psicologicamente saudáveis (Larson, 2000).

Como aconteceu no âmbito da saúde (Antunes, 2008), também na psicologia algumas abordagens teóricas e empíricas procuram superar as "lentes do deficit" centradas na fragilidade e na doença (Benson, 2007). É o caso da psicologia positiva (Seligman \& Csikszentmihaly, 2000) e, partilhando a ideia de que a prevenção de problemas não garante o desenvolvimento positivo nem uma preparação adequada para a vida adulta (Small \& Memmo, 2004), o "Positive Youth Development" (PYD).

Com origem nas teorias sistémicas do desenvolvimento (Silbereisen \& Lerner, 2007), o PYD 
centra-se nas forças, capacidades e possibilidades da pessoa integral e única de cada jovem, e nos elementos ou recursos existentes nos contextos em que este se desenvolve, valorizando a relação bidirecional e de mútua influência entre a pessoa e o seu contexto (Benson, 2007; Benson, Scales, Hamilton, Sesma, Hong \& Roehlkepartain, 2006; Larson, 2000).

Alguns autores identificaram dois desafios ao nível do PYD: (1) a multiplicidade de operacionalizações, indicadores de bem-estar, de comportamentos de risco, desenvolvimento positivo e recursos de desenvolvimento (Scales, 2014); (2) a escassez de instrumentos para a avaliação do desenvolvimento positivo e do bem-estar na adolescência (Fernandes, 2007).

Várias propostas procuram responder a estes desafios. É o caso dos $6 \mathrm{Cs}$ - competência, confiança, conexão, carácter, cuidado e contribuição (Silberreisen \& Lerner, 2007) e, no centro deste estudo, os "desevolpmental assets".

Os "desevolpmental assets", traduzidos neste estudo por Recursos de Desenvolvimento (RD) - procurando assegurar a expressividade do conceito e, ao contrário da opção de outros autores (e.g. Gutiérrez \& Gonçalves, 2013; Santos \& Gonçalves, 2012), evitando a difusão semântica de "ativos de desenvolvimento" - resultam da investigação em torno dos atributos pessoais e dos recursos dos contextos promotores de desenvolvimento (Eccles \& Gootman, 2002). Neste sentido os RD são "nutrientes" (Benson, 2007) ou "blocos de construção" que todas as crianças e adolescentes precisam para um desenvolvimento bem-sucedido (Scales, 1999).

Ao longo do tempo, estudos longitudinais com mais de três milhões de adolescentes e jovens norte-americanos, (Benson, Scales, \& Syvertsen, 2011; Search Institute, 2006) têm mostrado consistentemente uma associação de sinal positivo entre estas forças (pessoais ou internas e contextuais ou externas) e vários indicadores de bemestar e de desenvolvimento (sucesso escolar, comportamentos de ajuda informais, liderança, capacidade de resistir ao perigo, autocontrolo, valorização da diversidade, valorização de comportamentos saudáveis e capacidade de ultrapassar as dificuldades e adversidades), e de sinal negativo com problemas e comportamentos de risco (Benson, 2007; Benson et al., 2012; Benson \& Scales, 2014; Scales, 2011).

É neste contexto que o Search Institute, referência norte-americana no âmbito do PYD (e.g. Merrel \& Gueldner, 2010), a partir da sua atividade de investigação e do trabalho de Peter Benson, apresenta um quadro teórico de referência dos RD (Scales, 1999) conhecido como "Developmental Assets Framework" e um instrumento de avaliação conhecido como "Developmental Assets Profile" ou DAP.

O "Developmental Assets Framework", apresentado na Tabela 1, propõe quarenta $\mathrm{RD}$ organizados em duas categorias, recursos contextuais ou externos (RD-E) e recursos pessoais ou internos (RD-I), cada uma delas com quatro dimensões ou fatores como é possível ver ta Tabela 1 (Search Institute, 2005).

$\mathrm{Na}$ sequência deste marco teórico, respondendo à necessidade de instrumentos de avaliação com fortes propriedades psicométricas (Benson et al., 2011), surge em 2005 o DAP ("Developmental Assets Profile" ou em português Perfil de Recursos de Desenvolvimento, com presença destacada em várias revisões de instrumentos no âmbito do PYD (e.g. Haggerty, Elgin \& Woolley, 2011; Wilson-Ahlstrom, Yohalem, DuBois, Adler, Hillaker \& Weikart, 2014).

Além da investigação em torno da validade psicométrica do DAP nos EUA, os autores defendem a fiabilidade e sensibilidade transcultural do instrumento referindo adaptações em países como Albânia, Bangladesh, Japão, Líbano e Filipinas (Scales, 2011) onde encontraram propriedades psicométricas próximas das encontradas nos jovens americanos (Benson et al. 2011; Benson \& Scales, 2014). O presente artigo tem por base apresentar a adaptação do DAP à realidade de uma amostra de adolescentes portugueses.

\section{Método}

\section{Participantes}

A amostra deste estudo é formada por 201 alunos de uma escola particular de Lisboa, entre os 10 e os 18 anos $(\mathrm{M}=12,85)$, do sexo feminino $(42,8 \%)$ e masculino $(57,2 \%)$, e do $6^{\circ}$ ano $(52,2 \%), 9^{\circ}$ ano $(31,8 \%)$ e $11^{\circ}$ ano $(15,9 \%)$. Optou-se na seleção dos participantes por incluir os alunos das turmas em final de ciclo como é o caso do $6^{\circ}$ e $9^{\circ}$ ano e o $11^{\circ}$ ano pelas especificidades associadas ao $12^{\circ}$ (último ano do secundário).

\section{Instrumentos}

DAP. O "Developmental assets profile" foi desenvolvido pelo Search Institute (2005), sendo composto por 58 itens organizados em duas sub-escalas: recursos de desenvolvimento externos (RD-E); recursos de desenvolvimento internos (RD-I).

Como se pode ver na Tabela 1, os RD-E são compostos por 26 itens organizados em quatro dimensões e os RD-I por 32 itens também organizados em quatro dimensões.

A avaliação de cada item é feita através de uma escala tipo likert de 4 possibilidades ( 0 - Nunca ou raramente; 1 - Pouco ou às vezes; 2 - Muito ou frequentemente, 3 Completamente ou quase sempre).

Tabela 1.

DAP - Dimensões, itens e consistência interna original

\begin{tabular}{lcc}
\hline \multicolumn{1}{c}{ Escala } & Itens & $\alpha$ \\
\hline Total & 58 & .97 \\
\hline Externos (RD-E) & 26 & .94 \\
Suporte (SU) & 7 & .80 \\
Empowerment (EM) & 6 & .74 \\
Limites e expectativas (LE) & 9 & .84 \\
Uso tempo (UT) & 4 & .56 \\
\hline Internos (RD-I) & 32 & .92 \\
Compromisso aprendizagem & 7 & .83 \\
(CA) & 11 & .85 \\
Valores positivos (VP) & 8 & .79 \\
Competências sociais (CS) & 6 & .79 \\
Identidade positiva (IP) &
\end{tabular}


Sendo a primeira vez este instrumento foi adaptado a uma amostra portuguesa, pelo menos de forma reconhecida pelo Search Institute, foram assegurados um conjunto de procedimentos (Borsa, Damásio \& Bandeira, 2012): (1) Tradução especializada para português; (2) Discussão da versão traduzida com peritos onde vingou, face a alguns itens problemáticos, a fidelidade ao original; (3) Avaliação da compreensão dos itens com um grupo-teste de alunos do $7^{\circ}$ ano; (4) Aplicação-piloto com um segundo grupo de alunos do $7^{\circ}$ ano; (5) Realização especializada de uma retroversão e envio aos autores.

EBEP-Adolescentes. A "Escala de Bem-estar Psicológico para Adolescentes" foi construída no âmbito dos trabalhos de Carol Ryff. A versão utilizada resulta da adaptação realizada por Helder Fernandes (2007).

A escala original é constituída por 30 itens representando as 6 dimensões do bem-estar psicológico: (a) autonomia - AUT, (b) domínio do meio (-), (c) crescimento pessoal - CRP, (d) relações positivas com os outros - REP, (e) objetivos na vida - OBV, (f) aceitação de si - ACS.

A avaliação de cada item é feita através de uma escala tipo likert de 5 pontos $(1-$ discordo totalmente e 5 discordo plenamente). $\mathrm{Na}$ sequência da presente investigação e dos estudos de validade interna realizados, optou-se por utilizar uma versão reduzida da escala com 19 itens e apenas 5 dimensões (excluindo a dimensão Domínio do Meio).

\section{Procedimiento}

A partir do projeto da investigação assegurou-se a autorização dos autores das escalas, deu-se início à adaptação do DAP e à construção do questionário final em formato digital (aplicação Google docs) para o preenchimento online. Assegurou-se também a autorização das escolas, a informação e autorização dos pais e encarregados de educação (por email) e, assegurando o consentimento informado, o autor do estudo apresentou em cada turma o mesmo pedido aos alunos a sua participação voluntária. $\mathrm{Na}$ sequência do encontro com o grupo de $7^{\circ}$ e do estudo-piloto, realizouse a apicação dos inquéritos nos dias 05, 06 e 11 de Dezembro de 2013 em períodos de 30 minutos, sendo os participantes acolhidos na sala de informática pelo autor do estudo ou um elemento do gabinete de psicopedagogia (quando o preenchimento foi realizado em simultâneo).

Todos os alunos participaram de forma motivada. Além da motivação o formulário online permitiu reduzir erros de preenchimento. $\mathrm{O}$ tratamento e análise estatística realizou-se com recurso ao programa informático Statistical Package For Social Sciences (SPSS) versão 20.

\section{Resultados}

Os resultados apresentados têm por base a avaliação das propriedades psicométricas do DAP - Developmental Assets Profile (Perfil de Recursos de Desenvolvimento), nomeadamente o estudo da validade interna (análise fatorial, correlação entre dimensões e consistência interna) e o estudo de validade externa (correlação entre as dimensões do DAP e as dimensões da EBEPAdolescentes).

\section{Validade interna}

A proposta teórica dos Recursos de Desenvolvimento (RD), apresenta duas categorias ou subescalas, RDExternos (RD-E) e RD-Internos (RD-I), cada uma delas organizada em quatro dimensões ou fatores. Para perceber como estas dimensões, no seu conjunto, explicam a variância existente e, por outro lado, para aferir se estas dimensões ou fatores fazem sentido na amostra portuguesa, i.e., se a associação estatística entre os diferentes itens é suficientemente significativa para assumir que estão a medir a mesma dimensão e não outra (justificando as dimensões propostas), realizou-se uma análise fatorial, adotando a prática de outros estudos (Scales, 2011), de analisar separadamente a escala dos RD-E (26 itens) e a dos RD-I (32 itens)

A análise de componente principal com rotação ortogonal (Varimax) para as quatro dimensões originais, mostram que estas explicam $48 \%$ da variância total no cado dos RD-E e $40 \%$ da variância total no caso dos RDI. Na sequência da análise da saturação dos itens, optouse pela fidelidade à estrutura original, excluíram-se os itens que saturavam abaixo do valor de referência (.30) ou que saturavam numa dimensão (fator) que não a dimensão original.

A seleção resultou num total de 14 itens na escala dos RD-E e de 19 itens na escala dos RD-I. Cada escala voltou a ser submetida à mesma análise de componente principal (Varimax) para quatro dimensões sendo os resultados da variância e da saturação dos itens por dimensão, visíveis na Tabela 2 . 
Tabela 2.

Análise fatorial dos RD-E e dos RD-I

\begin{tabular}{|c|c|c|c|c|}
\hline RD-E & SU & LE & UT & EM \\
\hline Item 56. & ,791 & & & \\
\hline Item 54. & ,780 & & & \\
\hline Item $47 .$. & ,751 & & & \\
\hline Item 13. & ,666 & & & \\
\hline Item 51. & ,432 & & & \\
\hline Item 45 . & & 399, & & \\
\hline Item 44. & & ,787 & & \\
\hline Item 57. & & ,768 & & \\
\hline Item 50. & & ,710 & & \\
\hline Item 43. & & 630 & & \\
\hline Item 40 . & & & ,788 & \\
\hline Item 31. & & & ,728 & \\
\hline Item 17. & & & & ,794 \\
\hline Item 46. & & & & ,665 \\
\hline \% variância total & \multicolumn{4}{|c|}{58,166} \\
\hline $\begin{array}{c}\text { RD-I } \\
\end{array}$ & IP & $\mathrm{CA}$ & $\mathrm{CS}$ & $\mathrm{VP}$ \\
\hline Item 12. &, 721 & & & \\
\hline Item 3. & 681 & & & \\
\hline Item 2. & ,620 & & & \\
\hline Item 15. &, 583 & & & \\
\hline Item 27. &, 516 & & & \\
\hline Item 14. & ,498 & & & \\
\hline Item 7. & & 690 & & \\
\hline Item 10. & & ,625 & & \\
\hline Item 5. & & 624 & & \\
\hline Item 8. & & 604 & & \\
\hline Item 26. & & ,557 & & \\
\hline Item $38 .$. & & ,385 & & \\
\hline Item $20 .$. & & & ,730 & \\
\hline Item 39. & & & ,728 & \\
\hline Item 24. & & &, 550 & \\
\hline Item 11. & & & 477 & \\
\hline Item 6. & & & 370 & \\
\hline Item 23. & & & & ,756 \\
\hline Item 22. & & & & ,712 \\
\hline \% variância total & & & & \\
\hline
\end{tabular}

$\mathrm{Na}$ análise das correlações entre as dimensões do DAP verifica-se a existência de correlações estatisticamente significativas entre grande parte das dimensões dos RDE e dos RD-I. Os valores moderados das correlações, ver Tabela 3, permitem salvaguardar a existência de dimensões distintas.

Tabela 3.

Correlações entre as dimensões do DAP

\begin{tabular}{|c|c|c|c|c|c|c|c|c|}
\hline & \multicolumn{4}{|c|}{ RD-E } & \multicolumn{4}{|c|}{ RD-I } \\
\hline & $\mathrm{SU}$ & EM & LE & UT & $\mathrm{CA}$ & $\mathrm{VP}$ & $\mathrm{CS}$ & IP \\
\hline SU & 1,000 & & & & & & & \\
\hline EM &, $285^{* *}$ & 1,000 & & & & & & \\
\hline LE &, $483^{* *}$ &, $256^{* *}$ & 1,000 & & & & & \\
\hline UT &, $197^{* *}$ & $\mathrm{~ns}$ &, $152^{*}$ & 1,000 & & & & \\
\hline $\mathrm{CA}$ &, $363^{* *}$ & ns & $440^{* *}$ &, $267^{* *}$ & 1,000 & & & \\
\hline VP &, $222^{* *}$ & $\mathrm{~ns}$ & $\mathrm{~ns}$ & ns &, $243^{* *}$ & 1,000 & & \\
\hline $\mathrm{CS}$ &, $296^{* *}$ &, $143^{*}$ &, $412^{* *}$ &, $258^{* *}$ &, $256^{* *}$ & ,298* & 1,000 & \\
\hline IP &, $425^{* *}$ &, $192^{* *}$ &, $389^{* *}$ &, $195^{* *}$ &, $341^{* *}$ &, $333^{* *}$ &, $365^{* *}$ & 1,000 \\
\hline
\end{tabular}

Legenda: ${ }^{*} p<0.05 ;{ }^{* *} p<0.01 ;$ ns - não significativo
A análise da consistência interna foi realizada através do cálculo do Coeficiente Alfa de Cronbach. Os resultados, apresentados na Tabela 4 , mostram resultados bons para o total dos itens $(\alpha=.859)$, apesar de inferiores aos valores da aplicação-piloto e de referência originais $(\alpha=.94)$ (Search Institute, 2005)

Quando analisadas as subescalas RD-E e RD-I os resultados, igualmente abaixo dos valores da aplicaçãopiloto e de referência, podem ser considerados bons ( $\alpha$ $=.759$ e .789 respetivamente). Quanto aos valores para as quatro dimensões de cada subescalas, podem ser considerados promissores (Scales, 2011), à exceção dos das dimensões "empowerment" $(\alpha=.427)$ e uso do tempo ( $\alpha=.434)$, cujo baixo valor poderá ser justificado pelo reduzido número de itens (Fernandes, 2007; Field, 2009).

Tabela 4.

Correlações entre as dimensões do DAP

\begin{tabular}{lcc}
\hline \multicolumn{1}{c}{ Escala e dimensões } & Itens & $\alpha$ \\
\hline Total & 33 & .859 \\
Recursos externos (RD-E) & 14 & .759 \\
Suporte (SU) & 5 & .749 \\
Empowerment (EM) & 2 & .427 \\
Limites e expectativas (LE) & 5 & .752 \\
Uso tempo (UT) & 2 & .434 \\
\hline Recursos internos (RD-I) & 19 & .789 \\
Compromisso aprendizagem (CA) & 6 & .648 \\
Valores positivos (VP) & 2 & .600 \\
Competências sociais (CS) & 5 & .615 \\
Identidade positiva (IP) & 6 & .717 \\
\hline
\end{tabular}

\section{Validade externa}

O estudo da validade externa, ligado à legitimidade do instrumento, teve por base a análise das correlações das dimensões do DAP com as da EBEP-Adolescentes.

$\mathrm{Na}$ tabela 5 é possível identificar correlações estatisticamente significativas e positivas entre os valores globais das escalas, o que acontece com grande parte das dimensões. Verificam-se também, de forma consistente com a literatura, correlações estatisticamente significativas mas de sinal negativo, entre algumas das dimensões do DAP e a dimensão Autonomia da EBEP.

Estes valores reafirmam a relação existente na literatura entre os RD e o Bem-Estar Psicológico (Benson \& Scales, 2014; Eccles \& Gootman, 2002), e permitem validar o instrumento e as opções realizadas.

Tabela 5.

Correlações entre as dimensões do DAP e da EBEPAdolescentes

\begin{tabular}{lcccccc}
\hline & Aut & CrP & ReP & ObV & AcS & $\begin{array}{c}\text { BEP } \\
\text { Global }\end{array}$ \\
\hline RD-E &,$- 171^{*}$ &, $322^{* *}$ &, $452^{* *}$ & ns &, $434^{* *}$ &, $395^{* *}$ \\
\hline RD-I &,$- 146^{*}$ &, $345^{* *}$ &, $477^{* *}$ &, $274^{* *}$ &, $593^{* *}$ &, $558^{* *}$ \\
\hline RD &,$- 174^{*}$ &, $371^{* *}$ &, $516^{* *}$ &, $208^{* *}$ &, $576^{* *}$ &, $535^{* *}$ \\
\hline
\end{tabular}

Legenda: ${ }^{*} p<0.05 ;{ }^{* *} p<0.01 ;$ ns - não significativo 


\section{Discusión}

A solidez teórica e empírica do DAP, apresentada na revisão de estudos norte-americanos e realizado noutros países do mundo, não se verificou na presente amostra. Face à fragilidade psicométrica inicial, optou-se por uma seleção dos itens que permitiu um instrumento final com alguma qualidade, visível nas correlações estatisticamente significativas com as dimensões da EBEP-Adolescentes. São resultados que justificam um maior investimento na adaptação do instrumento.

Nesse sentido alguns comentários conclusivos podem ser úteis. O primeiro comentário diz respeito à fidelidade à proposta original como critério utilizado para orientar a adaptação do instrumento, incluindo a tradução, os procedimentos associados a itens problemáticos, as opções de resposta dos participantes e, face ao estudo da validade interna, a seleção dos itens. Santos \& Gonçalves (2012) na adaptação do DAP a uma amostra de jovens atletas portugueses, retiveram apenas 22 itens e 6 dimensões. Tendo assegurado, como foi feito neste estudo, a compreensão dos itens, os autores concluem que os itens do DAP tendem a refletir a cultura norteamericana. Noutros estudos deve refletir-se na importância de rever os itens à luz da cultura dos participantes, ou seja, sem comprometer a integridade conceptual, permitir alterações ponderadas aos itens e à sua formulação.

O segundo comentário diz respeito à especificidade da amostra, composta exclusivamente por alunos de uma escola particular. Apesar do interesse dos resultados e da sua relação com resultados de outros estudos ao nível do desenvolvimento de crianças e jovens, é importante em estudos futuros, a opção por amostras mais heterogéneas. O terceiro comentário, na linha do anterior, diz respeito à distribuição desigual da amostra, com um grande grupo de alunos no $6^{\circ}$ ano $(54,4 \%)$. É uma desigualdade que se reflete nos resultados gerais obtidos a partir de valores médios e que deve ser acautelada noutros estudos.

No sentido de responder ao desafio de uma abordagem que apoie a investigação e a intervenção no âmbito do desenvolvimento das crianças e dos jovens do século XXI, é importante uma atenção redobrada às diferentes propostas de forma a construir melhores indicadores e instrumentos para perceber os recursos pessoais, mas também os recursos contextuais promotores de um desenvolvimento positivo.

Nota: O presente estudo insere-se num outro mais amplo, dissertação de mestrado com o título "Bem-estar psicológico e recursos contextuais e pessoais em adolescentes de uma escola particular portuguesa", realizada pelo primeiro autor com a orientação do segundo autor, Professor Feliciano H Veiga.

\section{Referencias}

Antunes, M. C. P. (2008). Educação, saúde e desenvolvimento. Coimbra: Edições Almedina.

Benson, P. L, Scales, P. C., \& Syvertsen, A. K. (2011). The contribution of the developmental assets framework to positive youth development theory and practice. Advances in Child Development and Behavior, 41, 197-230.

Benson, P. L., \& Scales, P. C. (2014). Developmental Assets. In: R. J. R. Levesque (Ed.). Encyclopedia of Adolescence (pp. 667-683). New York: Springer.

Benson, P. L. (2007). Developmental assets: Na overview of Theory, research and practice. In R. K. Silbereisen \& R. M. Lerner. Approaches to Positive Youth Development (pp. 33-58). London: Sage Publications.

Benson, P. L., Leffert, N., Scales, P. C., \& Blyth, D. A. (2012). Beyond the "Village" Rhetoric: Creating Healthy Communities for Children and Adolescents. Applied Developmental Science, 16 (1), 3-23. DOI: 10.1080/10888691.2012.642771

Bizarro, L. (1999). O bem-estar psicológico durante a adolescência (dissertação de doutoramento em psicologia). Lisboa: Faculdade de Psicologia e Ciências da Educação da Universidade de Lisboa.

Borsa, J. C., Damásio, B. F., \& Bandeira, D. R. (2012). Adaptação e Validação de Instrumentos Psicológicos entre Culturas: Algumas Considerações. Paidéia, 22(53), 423-432.

Eccles, J., \& Gootman, A. (2002). Part 1: Promoting adolescente development. In Authors. Community Programs to Promote Youth Development (pp. 41118). Washington, D.C: National Research Council/ Institute of Medicine.

Fernandes, H. (2007). O bem-estar psicológico em adolescentes: uma abordagem centrada no florescimento humano (tese Doutoramento em Psicologia). Vila Real: Universidade de Trás-osMontes e Alto Douro.

Field, A. (2009). Discovering statistics Using SPSS (3rd Ed.). London: Sage Publications.

Gama, S. (2003). Diário: Pequena história da minha vida de professor. (12 ${ }^{\mathrm{a}}$ Ed.). Sintra: Edições Arrábida.

Gomez, B. J., \& Ang, P. M.-M. (2007). Promoting Positive Youth Development in Schools. Theory Into $\begin{array}{llll}\text { Practice, } & 46 & \text { (2), } & \text { 97-104. doi: }\end{array}$ $10.1080 / 00405840701232752$

Gutiérrez, M., \& Gonçalves, T. O. (2013). Activos para el desarrollo, ajuste escolar y bienestar subjetivo de los adolescentes. International Journal of Psychology and Psychological Therapy, 13 (3), pp. 339-355.

Larson, R. (2000). Toward a Psychology of Positive Youth Development. American Psychologist, 55 (1), 70-183.

Lerner, R. M. (2005). Promoting positive youth development: theoretical and empirical bases (White paper). Workshop on the Science of Adolescent Health and Development, National Research Council. Washington, DC: National Academy of Sciences.

Merrell, H. W., \& Gueldner, B. A. (2010). Social and Emotional Learning in the Classroom. Promoting mental health and academic success. New York: The Guilford press.

Santos, A. J., \& Gonçalves, C. E. (2012). Tradução do sources of enjoyment in youth sport questionnaire e do developmental assents profile para jovens atletas portugueses. (Ed.). Annals of research in sport And 
physical activity (pp. 9-36). Coimbra: Imprensa da Universidade de Coimbra.

Scales, P. C. (1999). Reducing risks and building developmental assets: Essential actions for promoting adolescent health. Journal of School Health, 69 (3), 113-119.

Scales, P. C. (2011). Youth developmental assets in global perspective: Results from international adaptations of the Developmental Assets Profile. Child Indicators Research, 4, 619-645. DOI: 10.1007/s12187-011-9112-8

Scales, P. C. (2014). Developmental Assets and the Promotion of Well-Being in Middle Childhood. In: A. Bem-Arieh, F. Casas, I. Frones \& J. E. Korbin (Eds.). Handbook of Child Well-Being (pp.1649-1678). Netherlands: Springer.

Search Institute (2005). Developmental Assets Profile: User manual. Minneapolis: Search Institute

Search Institute (2006). 40 Developmental Assets for Adolescents (ages 12-18). Minneapolis: Search Institute. Retirado de www.search-institute.org

Search Institute (2013). Developmental Assets Profile: Technical summary. Minneapolis: Search Institute

Seligman, M., \& Csikszentmihaly, M. (2000). Positive psychology: An introduction. American Psychologist, 5 (1), 5-14. DOI 10.1037/0003-066X.55.1.5.

Silbereisen, R. K., \& Lerner, R. M. (Eds.) (2007). Approaches to positive youth development (pp. 3-30). London: Sage Publications.

Simões, M. C. R. (2007). Comportamentos de risco na adolescência. Lisboa: Fundação Calouste Gulbenkian

Small, S., \& Memmo, M. (2004). Contemporary models of youth development and problem prevention: Toward an integration of terms, concepts, and models. Family Relations, 53, 3-11. 